\section{Case Reports in Orthopedic Research}

Case Rep Orthop Res 2020;3:118-122

\title{
Physical Manipulation-Induced Femoral Neck Fracture: A Case Report
}

\author{
Rami A. Ayoubi Nour S. Nassour Elias G. Saidy Dany K. Aouad \\ Joseph S. Maalouly Elie B. Daou \\ Department of Orthopedic Surgery, Saint George Hospital Medical Center, University of \\ Balamand, Beirut, Lebanon
}

\section{Keywords}

Physical manipulative treatment · Hip fractures - Case report · Osteopathy · Chiropractic manipulation · Complication

\begin{abstract}
Manipulative treatments for pain are very widely used nowadays by a variety of physicians. These treatment modalities are generally safe, but various studies have reported serious complications. This report presents the case of a 78-year-old male patient with a history of Parkinson's disease, who was diagnosed with a right hip fracture that occurred as a result of physical manipulative treatment. He underwent a cemented hemi-arthroplasty as appropriate surgical treatment of his condition. Manipulative treatments can result in minor and major complications ranging from simple sprains and rib fractures to cerebrovascular accidents and death. The frequency of these events seems to be rare; however, no robust studies are present and further investigations are urgently needed. Hip fractures as a result of this treatment have not been previously mentioned. This is the first reported case in the literature of a hip fracture resulting from manipulative treatment. Primary care physicians and orthopedists should be aware of the possibility of this outcome and rule it out whenever necessary.
\end{abstract}




\section{Case Reports in Orthopedic Research}

\section{Introduction}

Manipulative treatments have been very popular as a treatment option of nonspecific muscle aches, especially neck and low back pain. These manipulations are manual and noninvasive procedures, mostly done by chiropractors, osteopathic physicians, physiotherapists, physicians, and other health-care providers [1]. However, since this practice involves the use of physical force, it can have unwanted consequences, especially if the patient is prone to injury, the provider has inadequate skills, or the treatment is improperly applied [2]. In a retrospective review, Vick et al. [3] mention the many injuries that can be sustained by patients undergoing such manipulative treatments; these can include, but are not limited to, bone fractures or dislocations, general increase in pain, disc herniation, and even cerebrovascular incidents [3]. It is worthwhile noting that these injuries are generally reported following a chiropractic session involving forceful high-velocity movements [3]. According to our knowledge, cases of femoral neck fractures as a result of manipulative treatments have never been previously reported.

In this report, we are presenting the case of a 78-year-old male patient who sustained a femoral neck fracture following osteopathic manipulation.

\section{Case Presentation}

This is the case of a 78-year-old male patient, known to have Parkinson's disease, initially presenting to the hospital for management of anemia. The orthopedic surgery department was consulted for right hip pain and swelling. His past medical history includes coronary artery disease, chronic kidney disease, obstructive sleep apnea, bilateral deep vein thrombosis, and hypertension.

These symptoms had started following a spinal manipulation treatment 1 month prior to presentation. At the time, he was undergoing osteopathic spinal manipulation treatment for low back pain radiating bilaterally to his lower limbs, which includes manipulation of the hips as part of the treatment regimen. After the last session that was conducted, he reported pain over his right hip area, and he refused to ambulate afterwards. However, as the patient had multiple comorbidities and his baseline ambulation was minimal, the family did not pay any particular attention to this. The following week, his primary care physician was consulted for the hip pain, and an initial diagnosis of trochanteric bursitis was supposed, for which he received a local corticosteroid injection. In the following weeks, the patient's general status deteriorated with increased fatigue and a persistent inability to ambulate. An outpatient laboratory workup was done that showed severe anemia.

As a result, the patient was admitted to our hospital for management of anemia. The orthopedic surgery team was consulted at the time of admission for evaluation of right hip pain and decreased ambulation. The bedside physical exam of the right hip revealed a painful and decreased range of motion, with tenderness to palpation over the right greater trochanter. The patient refused to ambulate at the time. The history related to this pain was collected from the patient's wife, who denied any history of trauma or falls, as his capacity to communicate was affected by his Parkinson's disease. Radiographs of the right hip were obtained and revealed a chronic femoral neck fracture with the femoral head identified in the acetabulum with ascension of the femur proximal to the level of the acetabulum (Fig. 1). The patient's family reported that he could minimally ambulate with help from another person, so a decision to 


\section{Case Reports in Orthopedic Research}

Case Rep Orthop Res 2020;3:118-122

DOI: 10.1159/000509704

(c) 2020 The Author(s). Published by S. Karger AG, Basel www.karger.com/cio

Ayoubi et al.: Physical Manipulation-Induced Femoral Neck Fracture: A Case Report

proceed with cemented hemiarthroplasty, as an appropriate surgical treatment of his condition, was taken.

The procedure was well tolerated, no peri-operative complications occurred, and the operative radiographs are shown in Figure 2. Postoperatively, the patient had no complications, assisted ambulation with weightbearing as tolerated was begun on day 1 postoperatively, and the recovery was smooth.

\section{Discussion}

Injuries resulting from manipulative treatments, especially spinal manipulation, have been previously reported in several case reports and case series and have been investigated in systematic reviews [2]. There is still no consensus on the frequency of serious accidents attributable to manipulative treatment, with a study reporting this risk to be 1.3 cases over 100,000 persons and a systematic review reporting this frequency to vary from 0.05 to 1.46 per 10,000,000 manipulations $[4,5]$.

Manipulative treatments generally seem to be a nonharming modality of treatment; however, complications, such as radiculopathy, myelopathy, cerebrovascular accidents, and even fracture dislocation of the cervical spine, have been previously reported [6]. Vick et al. [3], in their review of the literature conducted in 1996, divided the resulting complications into minor (sprains and rib fractures) and major (cerebrovascular accident, neural insult, major bone fracture or dislocation, and disc disease). Rinsky et al. [7] mentioned that various neurological complications can result as a consequence of chiropractic manipulation, such as injuries to the brainstem, spinal cord, cauda equina, or the brachial plexus; even death has been seldom reported $[7,8]$. Head and neck manipulation can produce a particularly morbid complication, which is basilar artery thrombosis causing brain stem infarction and conceivably death in a high percentage of cases [7]. Guidelines for physicians involved in this type of manipulations recommended exercising caution when treating patients with coagulation defects, inflammatory spondyloarthropathies, osteoporosis, aortic dissection and aneurisms, or long-term usage of anticoagulant therapies [2].

Overall, physical manipulative treatments seem to have low rates of complications [4, 5]. However, some of the complications, although rare, are very serious in nature and can ultimately result in death $[7,8]$. Moreover, there is no consensus nor robust data concerning the prevalence of these critical complications and further studies are urgently needed to have a proper understanding of the rate of these incidents [5].

Unfortunately, during the hospitalization, the patient did not have a laboratory workup for vitamin D levels assessment, which is a risk factor for hip fractures. Ramason et al. [9] prospectively evaluated 485 elderly patients being admitted for hip fractures: $57.5 \%$ had vitamin D deficiency and 34.5\% vitamin D insufficiency, with only $8 \%$ of patients with hip fractures having sufficient vitamin D levels.

There have been no previous publications on hip fractures resulting from osteopathic or chiropractic manipulation. It is conceivable that this complication has previously occurred but has not been reported or that it has been misdiagnosed at the time. So, this case presents the noteworthy first reported case of a femoral neck fracture as a result of physical manipulative treatment. 


\section{Case Reports in Orthopedic Research}

\section{Conclusion}

As a conclusion, forceful manipulation during physical manipulative treatments can result, among numerous complications, in spinal fracture, dislocations, and, as presented in this case report, in a femoral neck fracture. As a result, primary care physicians and orthopedists should be aware, when examining elderly patients presenting with hip pain after a manipulative therapy session, of the possibility of the presence of a femoral neck fracture even without the occurrence of a fall or other traumas.

\section{Statement of Ethics}

Approval and consent of the ethics committee has been received for the publication of this article. Subjects (or their parents or guardians) have given their written informed consent to publish their case (including publication of images). No identifiers are included in this article relating to patient identity.

\section{Conflict of Interest Statement}

The authors declare that they have no competing interests.

\section{Funding Sources}

No funds were received in support of this study.

\section{Author Contributions}

R.A.A.: writing and editing the article. N.S.N.: writing and editing the article. E.G.S.: writing and editing the article, with editing of the images and radiographs. D.K.A.: writing and reviewing the article along with the referencing. J.S.M.: Writing and finalizing the article. E.B.D.: Surgeon who did the surgical procedure, writing and finalizing the article.

\section{Availability of Data and Material}

The datasets used and/or analyzed during the current study are available from the corresponding author on reasonable request.

\section{References}

1 Stevinson C, Ernst E. Risks associated with spinal manipulation. Am J Med. 2002 May;112(7):566-71.

2 Whedon JM, Mackenzie TA, Phillips RB, Lurie JD. Risk of traumatic injury associated with chiropractic spinal manipulation in Medicare Part B beneficiaries aged 66 to 99 years. Spine. 2015 Feb;40(4):264-70.

3 Vick DA, McKay C, Zengerle CR. The safety of manipulative treatment: review of the literature from 1925 to 1993. J Am Osteopath Assoc. 1996 Feb;96(2):113-5. 
Case Reports in Orthopedic Research
Case Rep Orthop Res 2020;3:118-122

(c) 2020 The Author(s). Published by S. Karger AG, Basel www.karger.com/cio

4 Rothwell DM, Bondy SJ, Williams JI. Chiropractic manipulation and stroke: a population-based case-control study. Stroke. 2001 May;32(5):1054-60.

5 Gouveia LO, Castanho P, Ferreira JJ. Safety of chiropractic interventions: a systematic review. Spine. 2009 May;34(11):E405-13.

6 Liao CC, Chen LR. Anterior and posterior fixation of a cervical fracture induced by chiropractic spinal manipulation in ankylosing spondylitis: a case report. J Trauma. 2007 Oct;63(4):E90-4.

7 Rinsky LA, Reynolds GG, Jameson RM, Hamilton RD. A cervical spinal cord injury following chiropractic manipulation. Paraplegia. 1976 Feb;13(4):223-7.

8 Pratt-Thomas HR, Berger KE. Cerebellar and spinal injuries after chiropractic manipulation. J Am Med Assoc 1947 Mar;133(9):600-3.

9 Ramason R, Selvaganapathi N, Ismail NH, Wong WC, Rajamoney GN, Chong MS. Prevalence of vitamin D deficiency in patients with hip fracture seen in an orthogeriatric service in sunny Singapore. Geriatr Orthop Surg Rehabil. 2014 Jun;5(2):82-6.

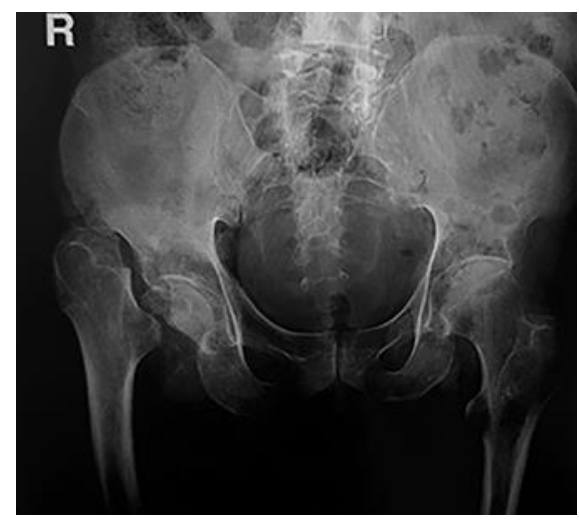

Fig. 1. AP pelvic X-ray showing a chronic right femoral neck fracture with proximal migration of the femur.

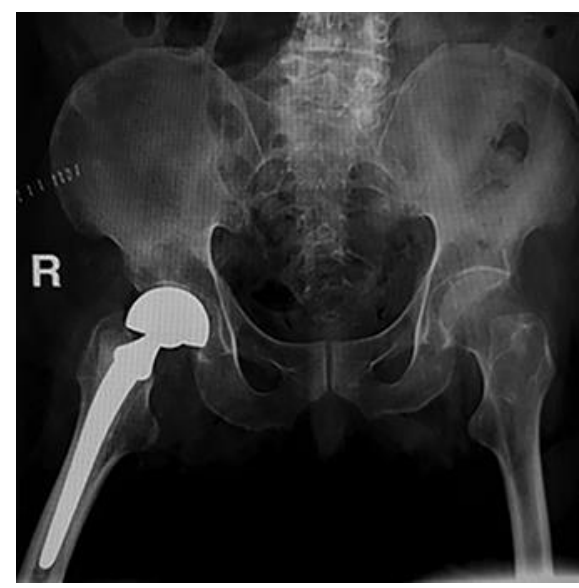

Fig. 2. Postoperative AP pelvis X-ray showing a right hemi-arthroplasty with a cemented femoral stem. 